\title{
Controlled tungsten melting and droplet ejection studies in ASDEX
}

\section{Upgrade}

K. Krieger, T. Lunt, R. Dux, A. Janzer, H.W. Müller, S. Potzel, T. Pütterich, Z. Yang and the ASDEX Upgrade Team

Max-Planck-Institut für Plasmaphysik, Garching, Germany

PACS: 28.52.Fa, 28.52.Nh, 52.25.Vy, 52.40.Hf, 52.70.Nc

Corresponding author address: Max-Planck-Institut f. Plasmaphysik, Boltzmannstr.2, 85748 Garching, Germany

Corresponding author email: krieger@ipp.mpg.de 


\begin{abstract}
Tungsten rods of $1 \times 1 \times 3 \mathrm{~mm}$ were exposed in single $\mathrm{H}$-mode discharges at the outer divertor target plate of ASDEX Upgrade using the divertor manipulator system. Melting of the W rod at a pre-defined time was induced by moving the initially far away outer strike point close to the W-rod position. Visible light emission of both the W-pin and consecutively ejected W droplets were recorded by two fast cameras with crossed viewing cones. The time evolution of the local W-source at the pin location was measured by spectroscopic observation of the WI line emission at $400.9 \mathrm{~nm}$ and compared to the subsequent increase of the tungsten concentration in the confined plasma derived from tungsten vacuum UV line emission. Combining these measurements with the total amount of released tungsten due to the pin melt events and ejected droplets allowed to derive an estimate of the screening factor for this type of tungsten source. The resulting values of the tungsten divertor retention in the range from 10-20 agree with those found in previous studies using a $\mathrm{W}$-source of sublimated $\mathrm{W}(\mathrm{CO})_{6}$ vapour at the same exposure location. Ejected droplets were found to be always accelerated in general direction of the plasma flow, attributed to friction forces and to rocket forces.

Furthermore, the vertically inclined target plates cause the droplets, which are repelled by the target plate surface potential due to their electric charge, to move upwards against gravity due to the centrifugal force component parallel to the target plate.
\end{abstract}




\section{Introduction}

Tungsten will be used in ITER for divertor and baffle plasma facing surfaces because of its high sputtering resilience, low $\mathrm{T}$ fuel uptake and favourable thermo mechanical properties. Safety limits with respect to tritium retention require furthermore the replacement of the initially used carbon fibre composite target plates towards a full tungsten divertor before the start of plasma operation with pure D and D-T fuel mix. Despite the high melting point of tungsten, off-normal transient heat loads in the divertor are still expected to exceed the thermo mechanical limits causing local surface melting of tungsten armour. If the resulting melt layers detach by boiling and/or electro mechanic forces, tungsten will be ejected into the divertor plasma in form of molten $\mathrm{W}$ droplets. The main question connected to $\mathrm{W}$ droplet ejection are, what fraction of the ejected molten material will become ionised and what fraction of the corresponding $\mathrm{W}$-source will in turn penetrate the confined plasma, which is of critical importance for the performance and safety of plasma operation. An additional issue is the distribution pattern of surviving droplets, because of their potential to cause additional surface damage at those locations where they eventually end up. First experimental studies on the behaviour of liquid tungsten ejected into the divertor plasma by means of controlled tungsten melt events in the divertor of ASDEX Upgrade were presented in [1]. Adding a second, tangentially oriented, fast camera system to the original setup with a vertical view down into the divertor, allowed to resolve the full trajectory of ejected droplets and to identify the dominant forces, which determine the motion of the droplets.

\section{Experimental setup and procedure}

For the tungsten ejection experiments a probe head equipped with a $\mathrm{W}$-pin of $1 \times 1 \mathrm{~mm}^{2}$ crosssection protruding $3 \mathrm{~mm}$ above the plasma facing surface was constructed for the ASDEX Upgrade divertor manipulator system (Fig. 1). The probe exposure position is located at the 
outer divertor target plate in the middle of divertor sector 2. Plasma flux and temperature were measured by flush mounted Langmuir probes [2] in sector 8 (i.e. $135^{\circ}$ toroidally from the pin position) while the power flux to the target plate was measured by a thermography camera system [3]. Spectral line emission in the visible range at the sample exposure location was detected by an array of optical fibres (Fig. 1) viewing the sample surface from below the dome baffle with the attached spectrometer tuned to the wavelength range around the tungsten WI line at $400.9 \mathrm{~nm}$ [4]. For the experiment discussed here, the W-pin sample was exposed in a H-mode discharge, \#26751, with plasma current $I_{p}=0.8 \mathrm{MA}$, toroidal field $B_{t}=-2.5 \mathrm{~T}$, average density $\bar{n}_{e}=7 \times 10^{19} \mathrm{~m}^{-3}$ similar to the experiment discussed in ref. [1], however with increased neutral beam heating power of $P_{N B I}=10 \mathrm{MW}$. Central electron cyclotron resonance heating with $P_{E C R H}=1 \mathrm{MW}$ for control of central W concentration failed before the first melting events but proved to be unnecessary as the steady state W-concentration did not increase over the value in the previous reference discharge, \#26750. Before the onset of auxiliary heating the outer strike point (OSP) was shifted $\approx 9 \mathrm{~cm}$ below the $\mathrm{W}$-pin position. Melting of the pin was initiated after full NBI power of $P_{N B I}=10 \mathrm{MW}$ was established at $t \simeq 1.9 \mathrm{~s}$ by moving the strike point upwards to $\approx 2 \mathrm{~cm}$ below the W-pin position (Fig. 2 ). At the end of the plasma flat top phase a sweep of the plasma across the installed Langmuir probes was performed to determine poloidal profiles of $n_{e}$ and $T_{e}$ at the outer target plate (Fig. 3). The parallel power flux at the pin location averaged over the time scale of ELM repetition ( $\Delta t_{E L M} \approx 8 \mathrm{~ms}$ ) was estimated to $\bar{q}_{\|} \approx 75 \mathrm{MWm}^{-2}$ from corresponding local values of $T_{e, i}=19 \mathrm{eV}$ and $n_{e}=7.5 \times 10^{19} \mathrm{~m}^{-3}$.

For the observation of $\mathrm{W}$ droplets ejected from the melting $\mathrm{W}$-pin into the divertor plasma, two fast visible range camera systems (frame rate $20000 \mathrm{fps}$ ) were installed. Figure 4 shows 
views into the vessel from the respective camera ports computed from the 3D CAD model of the ASDEX Upgrade vessel interior [5]. The transparent white overlays denote the cameras' field of view. One camera with a resolution of $256 \times 256$ pixels was mounted at the vessel top viewing vertically down to the exposure position (Fig. 4a) with its field of view spanning approximately 3 of the 16 toroidal sectors of the vessel. The second camera system with a resolution of $512 \times 512$ pixels was installed at a mid-plane main chamber port viewing tangentially towards the exposure position (Fig. 4b). A direct view of the tangential camera to the exposure position is shadowed by the inner divertor baffle but W-droplets moving downstream will come into view at $\approx 20^{\circ}$ toroidal distance from the $\mathrm{W}$-pin (Fig. 4a). To improve contrast of the droplet light emission against the bright plasma background light in the divertor, both cameras were equipped with a $\mathrm{H}_{\alpha} / \mathrm{H}_{\beta}$ rejection filter.

\section{Experimental results}

The movie taken with the downward viewing camera shows the W-pin glowing brightly already during the ramping-up phase of the NBI heating. Ejection of W droplets started at $t \simeq 2.0 \mathrm{~s}$ when the strike point closed in to $\approx 6 \mathrm{~cm}$ distance below the $\mathrm{W}$-pin. By computing an overlay of subsequent camera frames, the trajectories of ejected droplets can be visualised. The overlays were computed by extracting single frames in $2 \mathrm{~ms}$ time steps from the movies and normalising each frame with its average brightness to reduce distortion by bright frames during ELMs. Then the maximum value of each pixel over all frames in the given time interval was taken to construct the overlay image. Figure 5 shows the results for the time interval from $t=2.25-2.36 \mathrm{~s}$ with the camera frame overlays projected in the respective viewing areas within the 3D CAD representation of the vessel shown in Fig. 4. Both at the vertical (a) and tangential (b) camera view ejection of single droplets from the pin location is seen, which move in plasma downstream direction (Fig. 5a). The photo of a just partially 
molten W-pin after plasma exposure in Fig. 6 confirms that already before droplet release melting material moves toroidally in the direction of the plasma flow. This indicates that plasma friction is the dominant force acting on the tungsten melt, causing eventually release of melt droplets. Apart from friction, the radial thermal electron emission current with the toroidal magnetic field will produce a corresponding Lorentz force directed upwards against gravity. However, while these forces influence the motion of melt layers on a plasma exposed surface [6] they have no apparent effect on the W-pin.

As already reported in [1] based on only the vertical camera view (Fig. 5a), ejected droplets move initially toroidally along the target plate but after some time start to move radially outwards. This means that the droplets must at the same time move vertically upwards, which can now be directly observed by the tangentially viewing camera system (Fig. 5b). From the available geometry information of the cameras' optical systems expressed in 3D vessel coordinates, the trajectory of the droplets can be computed in the vessel coordinate system. For each camera pixel $\left(i_{x}, i_{y}\right)$ there is a corresponding viewing chord described by a straight line from the focus of the optics $\vec{P}_{0}$ at the camera side to the computed intersection with the viewed vessel surface, $\vec{P}_{1}\left(i_{x}, i_{y}\right)$. Hence, using two camera views, the two observation lines for a given droplet can be combined to compute the droplet position as the centre coordinate of the shortest connecting line between the two observation lines. The length of this line provides a measure for the systematic experimental error of the derived position due to the slight mismatch between the true camera views and their representation in the $3 \mathrm{D}$ vessel model. From a first qualitative analysis of the droplet trajectories one can already infer that the droplets are gliding over the target plate surface. Their distance to the surface is apparently of the same magnitude as surface position variations of the installed tiles because 
for a few droplets collisions with tile edges are observed, which result in splashing of the droplet in several smaller parts.

Taking into account that the vertical projection of the particle trajectories is typically a factor of 10 smaller than their horizontally travelled distance, the distance of the droplet from the W-pin in the horizontal plane as function of time was derived in [1] by projecting the line of sight between camera observation point and droplet into the horizontal plane through the Wpin. The acceleration of the droplets in this plane was obtained by taking the second time derivative of the travelled distance revealing average values of $\approx 400 \mathrm{~ms}^{-2}$ but decreasing over time from the initial value. Furthermore the motion of the droplets is not influenced by the passing of ELM filaments, presumably because the respective interaction time interval is too small to significantly affect the forces acting on the droplets. One should take into account, however, that this is only valid for typical ASDEX Upgrade ELM parameters and cannot be extrapolated to the larger ELM power densities as expected e.g. in ITER, even in the case of mitigated ELMs.

To obtain an estimate for the typical droplet mass and diameter, the total amount of lost molten mass was determined by weighing the W-pin pre- and post-exposure including resolidified residue at the sample surface. For the pin exposed in \#26751 the mass loss was $16.5 \mathrm{mg}$. Assuming that this mass were entirely contained in the 13 ejected droplets counted in the movies of \#26751, the average mass of a droplet would be $1.27 \mathrm{mg}$, corresponding to a diameter of $\approx 500 \mu \mathrm{m}$. As will be shown in the following section, this droplet size is in contradiction to the observed droplet trajectories, which suggest a smaller diameter.

\section{Forces on the $W$ droplets}

The time dependence of the observed toroidal and vertical droplet motion allows to draw conclusions on the forces acting on the droplets and their relative strength. In [1] it was shown 
that the droplet acceleration in toroidal direction is mainly due to plasma pressure forces and rocket forces, whereas electrostatic and Lorentz forces are orders of magnitude smaller for expected droplet sizes. However, it should be noted that the latter contributions become significant for the typically much smaller dust particles in the plasma boundary [7].

The plasma streaming around a droplet exerts a friction force in direction of the intersecting magnetic flux tube, which, taking into account that the velocity of the droplet is negligible against the plasma velocity, can be approximated by the force due to the plasma pressure difference between the upstream and downstream facing side of the droplet. Because the velocity of the droplet is small compared to the plasma flow velocity, one can consider the droplet as an immobile material target in the plasma. Using the expressions derived in [8] for the plasma density at the upstream and downstream sheath edge of such a target, $n_{e}^{\text {us }}$ and $n_{e}^{d s}$ respectively (assuming $M=0.4$ far away from the droplet) one obtains [1]

$$
\frac{F_{p}}{m}=\frac{\pi r^{2} \overbrace{\left(n_{e}^{u s}-n_{e}^{d s}\right)\left(k T_{e}+k T_{i}\right)}^{\Delta P}}{m_{W} \rho_{W} 4 \pi / 3 r^{3}} \approx \frac{1}{4 m_{W} \rho_{W}} \frac{n_{e}\left(k T_{e}+k T_{i}\right)}{r} .
$$

Apart from the friction force, an additional rocket force may act on the droplet also in direction of the intersecting magnetic flux tube because the corresponding parallel power flux reaches only the upstream facing side of the droplet. Provided the droplet does not rotate, the resulting temperature gradient in the droplet will lead to a net force due to the correspondingly inhomogeneous droplet ablation. The full solution of this problem for the surface ablation of a sphere with power flux to only one illuminated hemisphere is beyond the scope of this paper. However, an upper bound for the ablation force can be derived by approximating the droplet as thin plate. Following [1] the resulting rocket acceleration is

$$
\frac{F_{r}}{m}=\frac{3}{4 \sqrt{2 \pi} m_{W} \rho_{W}} \frac{p_{v a p}\left(T_{+}\right)-p_{v a p}\left(T_{-}\right)}{r}
$$


with $T_{+}$and $T_{-}$denoting the droplet temperature at upstream and downstream facing surface respectively. These temperatures can be computed from the total power balance at both surfaces. At the upstream facing surface the power flux $q_{\|}$received from the plasma is balanced by the power loss due to tungsten evaporation, thermal radiation and by heat transport to the downstream facing surface:

$$
q_{\|}=\Gamma_{v a p}\left(T_{+}\right) \frac{\Delta H_{v a p}}{N_{A}}+\varepsilon_{T} \sigma T_{+}^{4}+\frac{\kappa}{d}\left(T_{+}-T_{-}\right) .
$$

At the downstream facing surface we assume the power flux to the surface as negligible and hence

$$
0=\Gamma_{\text {vap }}\left(T_{-}\right) \frac{\Delta H_{\text {vap }}}{N_{A}}+\varepsilon_{T} \sigma T_{-}^{4}-\frac{\kappa}{d}\left(T_{+}-T_{-}\right) .
$$

The power loss by tungsten evaporation is given by the product of the specific evaporation heat per atom [9], $\Delta H_{\text {vap }} / N_{A}=824 \mathrm{kJmol}^{-1} / N_{A}$, with the tungsten evaporation flux $\Gamma_{\text {vap }}(T)=p_{\text {vap }}(T) / \sqrt{2 \pi m_{W} k_{B} T}$ where $m_{W}$ is the tungsten atomic mass and $p_{\text {vap }}(T)$ the tungsten vapour pressure at temperature $T$, which is derived from the vapour pressure $p_{\text {vap }}\left(T_{m}\right)$ at tungsten melt temperature $T_{m}$ [9] by the Clausius-Clapeyron relation

$$
p_{\text {vap }}(T)=p_{\text {vap }}\left(T_{m}\right) e^{-\frac{\Delta H_{\text {vap }}}{N_{A} k_{B}}\left(\frac{1}{T}-\frac{1}{T_{m}}\right)} .
$$

The radiation power loss from the droplet is given by the Stefan-Boltzmann law for a grey body radiator with $\varepsilon_{T}=0.347$ being the total emission coefficient of tungsten with respect to an ideal black body [10]. In the expressions for the heat flux from the upstream facing side to the downstream facing side of the droplet in eqns. $(3,4), \kappa$ denotes the thermal conductivity of tungsten. For the thickness $d$ of the thin plate approximation the droplet radius is taken. Because of the lack of data for molten tungsten, the value $\kappa=89.5 \mathrm{Wm}^{-1} \mathrm{~K}^{-1}$ measured at 
$3380 \mathrm{~K}[11]$ was used. For given parallel power flux $q_{\|}$the temperatures $T_{+}$and $T_{-}$can then be computed by solving the equation system (3) and (4).

The resulting droplet acceleration parallel to the magnetic field as a result of pressure, eq. (1), and rocket forces, eq. (2), for the local plasma parameters at the W-pin position (section 2) and assuming $T_{e}=T_{i}$ are plotted in Fig. 7a as function of droplet diameter. The acceleration by the rocket force is only weakly depending on droplet radius because, as shown in Fig. 7b, the temperature difference $T_{+}-T_{-}$and the corresponding difference of evaporation pressures is approximately proportional to the radius, which cancels out with the denominator in the right hand side of eq. (2). In contrast, the acceleration by plasma friction is inversely proportional to the droplet radius and therefore is significant mainly for small droplets, e.g. for the plasma parameters at the $\mathrm{W}$-pin location for $d \lesssim 0.1 \mathrm{~mm}$. Comparing the calculated acceleration to the measured average value of $\approx 400 \mathrm{~ms}^{-2}$ shows that the simple estimate of the droplet size from the W-pin mass loss and number of ejected droplets is about a factor of 10 too high. Consequently, one can conclude that during the ejection events a significant fraction of the material penetrates the divertor plasma not in form of a single droplet but as a more diffuse source, presumably in form of a fine spray. Such spray ejection could be directly observed in experiments at TEXTOR $[6,12]$ using a camera with a magnified view of an exposed tungsten melt layer sample.

Apart from the toroidal acceleration of ejected droplets with the plasma flow one also has to consider their vertical motion. This can be explained by considering the force balance on the droplets due to their motion along the target plate with vertical inclination angle $v$, shown in the diagram in Figure 8. Their toroidal acceleration in the horizontal plane is given by expressions (1) and (2) taking into account the angle $\theta$ of the local magnetic field against the horizontal plane 


$$
m a_{t}(s)=\left(F_{p}\left(n_{e}(s), T_{e}(s)\right)+F_{r}\left(n_{e}(s), T_{e}(s)\right)\right) \cos \theta .
$$

Both accelerating forces are functions of the local plasma density and temperature, which can be expressed as function of poloidal distance $s$ from the strike point along the target plate surface. As soon as the droplet moves toroidally along the target plate with velocity $v_{t}$, it is pulled radially outwards by the inertial force $F_{i}=v_{t}^{2}(t) / R(s)$ with $R(s)=R_{\text {sep }}+s \sin v$ the local major radius of the target plate at poloidal distance $s$ from the strike point. Additionally the droplet is accelerated vertically downwards by gravity $g$ and the vertical component of the acceleration by the plasma according to the local vertical inclination of the magnetic field $m a_{t}(s) \sin \theta$. The forces on the droplet in the vertical plane can be split into two components perpendicular and parallel to the target plate surface (see Figure 8). The net force pulling the droplet towards the surface is balanced by electrostatic repulsion between the target plate potential and the negative charge of the droplet due to its own sheath potential. Assuming the droplet acting as spherical capacitor one obtains

$$
\frac{F_{e}}{m}=\frac{Q E(d)}{m}=\frac{9 \varepsilon_{0}}{m_{W} \rho_{W}} \frac{T_{e}(s) E(d)}{r^{2}} \triangleq\left(\frac{v_{t}^{2}(t)}{R(s)} \cos v+\left(g+a_{t}(s) \sin \theta\right) \sin v\right) .
$$

The electrical field due to the sheath potential drop strongly increases towards the surface. As a result the droplet will always assume an equilibrium distance $d$ where the forces towards the surface are balanced against the electrostatic repulsion.

For the forces parallel to the target plate surface there is a downward component $F_{-}$due to gravity and plasma forces and an upward component $F_{+}$due to the inertial force. It should be noted, however, that this component is only directed upwards because of the radially outward vertical inclination of the ASDEX Upgrade outer target plate. For a target plate with opposite inclination, the respective component of the inertial force would point downwards. The acceleration of the droplet parallel to the target plate as a result of the sum of these forces is 


$$
a_{s}(t)=\frac{v_{t}^{2}(t)}{R(s)} \sin v-\left(g+a_{t}(s) \sin \theta\right) \cos v
$$

Because the local plasma parameters at the target plate vary poloidally across the target plate, the poloidal droplet position $s(t)$ appears implicitly in the right hand side of eq. (5) and also in the right hand side of eq. (7) by its dependency on $a_{t}(s(t))$. Furthermore, one has to take into account that the acceleration forces in eq. (5) depend on the radius of the droplet, which is decreasing with time by evaporation according to

$$
\frac{d r}{d t}=-\frac{\Gamma_{v a p}(T(s))}{\rho_{W}}
$$

with $T(s)=T\left(q_{\|}(s)\right)$ from eqns. $(3,4)$ also a function of $s(t)$. The resulting nonlinear system of differential equations $(5,7,8)$ was integrated numerically using the solver NDSolve implemented in the Mathematica software. Analytic expressions for the poloidal profiles of $n_{e}$ and $T_{e}$ along the target plate were obtained by fitting a functional form originally derived for divertor heat load profiles [13]

$$
f(s)=f_{B G}+\left(\frac{f_{\text {farsoL }}}{2}+\frac{f_{0}}{2} \exp \left(\left(\frac{s}{2 \lambda}\right)^{2}-\frac{s-s_{0}}{\lambda}\right)\right) \operatorname{erfc}\left(\left(\frac{S}{2 \lambda}\right)-\frac{s-s_{0}}{\lambda}\right)
$$

with free parameters $\lambda, s_{0}, S, f_{0}, f_{B G}, f_{\text {farSOL }}$ to the Langmuir probe data (Fig. 3). Figure 9 shows solutions of eqns. $(5,7,8)$ for several initial droplet diameters. Comparing the toroidal and poloidal trajectories to the observed droplet motion (Fig. 5) confirms again that the ejected droplets must be significantly smaller than the mass loss based estimate of $500 \mu \mathrm{m}$. The observed droplet trajectories are only compatible with initial droplet diameters of $\approx 60-100 \mu \mathrm{m}$. Smaller droplets evaporate so fast that they travel less than the observed toroidal distances before they vanish. Larger droplets fall down because their toroidal acceleration is too small so that the upward directed inertial force never exceeds gravity. 


\section{Droplet lifetime and divertor screening}

To determine the divertor screening of tungsten atoms originating from the $\mathrm{W}$-pin melt erosion, one has to relate the local $\mathrm{W}$ source to the resulting penetration of the confined main plasma by W ions escaping the divertor. Figure 10a shows the time evolution of the W-pin's integrated thermal light emission taken from movie of the vertical camera. The background light intensity in the vicinity of the pin was subtracted to suppress the additional light contributions from passing ELM filaments. The W-pin brightness is modulated by the ELMs with additional bursts, which can be directly correlated to ejection of droplets and are also always correlated with an ELM. The spectrometer line of sight viewing directly at the pin shows the intensity of the WI line emission at $400.9 \mathrm{~nm}$ corresponding to the local tungsten source (Figure 10b). In close correlation to the W-pin thermal emission the W-source shows small contributions due to ELMs and much stronger excursions during droplet ejection events. Following each of these bursts one observes a corresponding increase of the tungsten main plasma concentration (Figure 10c) over the stationary background level with a subsequent exponential decay on the tungsten confinement time scale. Possible contributions from the smaller W-pin source excursions by the ELMs cannot be separated from the much larger effect of the droplet ejection events. However, using the integral particle balance for tungsten ions in the confined main plasma, one can still obtain the average divertor screening factor $S_{d i v}$, which denotes the fraction of a divertor W source penetrating the confined plasma. The number of tungsten ions in the confined plasma, $N_{W}(t)$, is linked to the time dependent W-pin source, $\Phi_{W}(t) / S_{\text {div }}$, and the constant background W source, $\bar{\Phi}_{W}^{\text {core }}$, by the tungsten ion confinement time, $\tau_{W}$ :

$$
\frac{d N_{W}(t)}{d t}=\bar{\Phi}_{W}^{c o r e}+\frac{\Phi_{W}(t)}{S_{d i v}}-\frac{N_{W}(t)}{\tau_{W}} .
$$


From the measured time evolution of $c_{W}$ one can then derive the divertor screening factor by integrating eq. (10):

$$
\int_{t_{0}}^{t_{1}} \frac{\Phi_{W}(t)}{S_{d i v}} d t=\frac{1}{\tau_{W}} \int_{t_{0}}^{t_{1}}\left(N_{W}(t)-\bar{N}_{W}\right) d t
$$

where $\bar{N}_{W}$ is the stationary tungsten plasma content in the flat top phase. $N_{W}$ is approximated by $N_{W} \approx c_{W} \bar{n}_{e} V$ with a plasma volume $V=14 \mathrm{~m}^{3}$. The stationary tungsten content was taken from the equivalent reference discharge \#26750 without W-pin exposure and the central tungsten confinement time of $60 \mathrm{~ms}$ was derived from the exponential decay of $c_{W}$ after W emission from the pin ceased. The model for the droplet life time derived in [1] based on the local power balance of the droplet (3) yields a $20-40 \%$ fraction of tungsten evaporating from ejected droplets during their observed residence time in the divertor plasma, depending on droplet size. Taking this into account, one obtains from eq. (11) for \#26751 a divertor screening factor of $S_{d i v}=120$. In two additional exposures to similar plasma discharges the same analysis yields values in the same range of $S_{\text {div }}=165\left(\# 25623, P_{N B I}=10 \mathrm{MW}\right)$ and $S_{d i v}=200\left(\# 25514, P_{N B I}=5 \mathrm{MW}\right)$ respectively. By relating these values to the screening factor of a main chamber tungsten source $S_{S O L}=11$, which was determined in the same type of discharge using tungsten laser ablation [14], one obtains a divertor retention factor for the evaporated W atom fraction $R=S_{d i v} / S_{S O L}$ in the range of 10-20, which is similar to results obtained from studies using injection of sublimated $\mathrm{W}(\mathrm{CO})_{6}$ vapour [4].

\section{Conclusions}

Controlled tungsten melting experiments were for the first time carried out in a divertor tokamak with ITER relevant geometry and boundary plasma conditions. After heating a Wpin exposed at the outer target plate to melting temperature of tungsten, ejection of molten 
tungsten droplets was observed with typical lifetimes of $0.1 \mathrm{~s}$ and flight path lengths of $\mathrm{O}(1 \mathrm{~m})$. Analysis of the observed droplet trajectories shows that ejected droplets have a diameter in the range of $50-100 \mu \mathrm{m}$. The divertor retention of the evaporated fraction of the eroded W-pin was determined to $10-20$, similar to that of $\mathrm{W}$ atoms eroded at the target plates. The comparatively long survival time of ejected droplets and their long travelling distances in the divertor plasma have implications for ITER operation. On the one hand, droplets might splash at undamaged surface areas providing a seeding location for further surface deterioration. On the other hand, the comparatively small evaporation rate of the droplets will limit the corresponding source strength of $\mathrm{W}$ atoms penetrating the plasma. However, for quantitative extrapolation of both surface damage and tungsten penetration to the core plasma following melt ejection, the influence of the much higher expected ELM power and particle flux and the longer ELM repetition time in ITER must be taken into account by the respective models. 


\section{References}

[1] K. Krieger et al., J. Nucl. Mat. DOI 10.1016/j.jnucmat.2010.12.229.

[2] M. Weinlich et al., Europhysics Conference Abstracts (Proceedings of the 23rd EPS Conference on Controlled Fusion and Plasma Physics), Kiev, 1996, European Physical Society, Vol. 20 G/Part II, pp. 715-718.

[3] A. Herrmann et al., Plasma Physics and Controlled Fusion 37 (1995) 17.

[4] A. Geier et al., Plasma Phys. Control. Fusion 44 (2002) 2091.

[5] T. Lunt et al., Nucl. Instr. and Methods in Phys. Res. A623 (2010) 812.

[6] J.W. Coenen et al., J. Nucl. Mater. (2010), doi:10.1016/j.jnucmat.2010.09.046

[7] S. I. Krasheninnikov et al., Physics of Plasmas 11 (2004) 3141.

[8] T. Lunt et al., Plasma Phys. Control. Fusion 49 (2007) 1783.

[9] E.R. Plante et al., J. of Research of the Nat. Bureau of Standards 77A (1973) 237.

[10] R. Allen et al., J. Appl. Phys. 31 (1960) 1382.

[11] Y. S. Touloukian et al., Thermophysical Properties of Matter, Volume 1, (1970), IFI/Plenum Publishing, New York, p. 428.

[12] J. Coenen et al., this conference.

[13] T. Eich et al., Inter-ELM power fall-off length for JET and ASDEX Upgrade: measurement and comparison with heuristic drift-based model, submitted to Phys. Rev. Lett.

[14] T. Lunt et al., J. Nucl. Mater. (2010), doi:10.1016/j.jnucmat.2010.11.009. 


\section{Figure captions}

Figure 1: Poloidal cross-section through the ASDEX Upgrade vessel with divertor manipulator system and plasma configuration during the stationary phase of the $\mathrm{W}$-pin melt experiments. The enlarged cross-section of the outer divertor shows the viewing chords of a local glass fibre array for spectroscopic observation of the sample surface in the visible range.

Figure 2: Time traces of key plasma parameters and of the outer strike point (OSP) position at the target plate in discharge \#26751. The dashed part of $P_{E C R H}$ denotes the programmed schedule, which was interrupted at $t=1.44 \mathrm{~s}$. The photo of the $\mathrm{W}$-pin sample shows the relative position of the OSP to the W-pin. Also indicated is the time window of the OSP sweep to obtain Langmuir probe (LP) profiles of local ion flux and electron temperature at the outer target plate (see Fig. 3).

Figure 3: Profiles of $T_{e}$ and $n_{e}$ at the outer target plate as function of distance $\bar{s}$ from the strike point measured by Langmuir probes during a vertical sweep of the plasma across the probes. The nominal strike point position $(\bar{s}=0)$ during the flat top phase was $\approx 17 \mathrm{~mm}$ below the W-pin.

Figure 4: Field of view for the fast camera viewing from a top vessel port down into the divertor (a) and for the camera viewing from a mid-plane port tangentially towards the sector of the divertor manipulator (b).

Figure 5: Overlay of camera frames showing the trajectories of droplets following an ejection event at $t=2.25 \mathrm{~s}$ in $\# 26751$ for the vertical camera view (a) and the tangential camera view (b) respectively. The arrows indicate the position where the initially ejected single droplet split in two by a collision with the target plate surface. The droplet traces inside the circle in view (b) are ghost images of the original droplets created in the camera optics. 
Figure 6: Photo of W-pin exposed in discharge \#24997 without moving the strike point close to the pin. The plasma flux to the pin was sufficient to melt the tip of the pin resulting in molten material moving in toroidal direction due to friction with the incident plasma.

Figure 7: a) Acceleration of droplets due to plasma friction and rocket forces calculated as function of droplet diameter for local plasma conditions at the W-pin location in discharge \#26751. b) Temperature difference between plasma exposed and shadowed side of a Wdroplet as function of droplet diameter for local plasma conditions at the W-pin location in discharge \#26751.

Figure 8: Forces in the poloidal plane acting on a $\mathrm{W}$ droplet sliding over the target plate surface.

Figure 9: Vertical motion (a) and toroidal motion (b) of a W droplet as result of local force balance computed for local plasma parameters during flat-top phase of \#26751 for different initial droplet diameters. The coordinate $\bar{s}$ in (a) denotes the poloidal distance to the nominal strike point position along the outer target plate. The coordinate $x$ in (b) denotes the toroidal distance from the W-pin position. (c) shows the decrease of droplet diameter with time due to evaporation normalised to the initial diameter at droplet release.

Figure 10: Time traces of integrated visible light intensity of the W-pin (a), local tungsten source at the W-pin position from WI line intensity (b) and tungsten main plasma concentration (c) in discharge \#26751. 
Figure 1

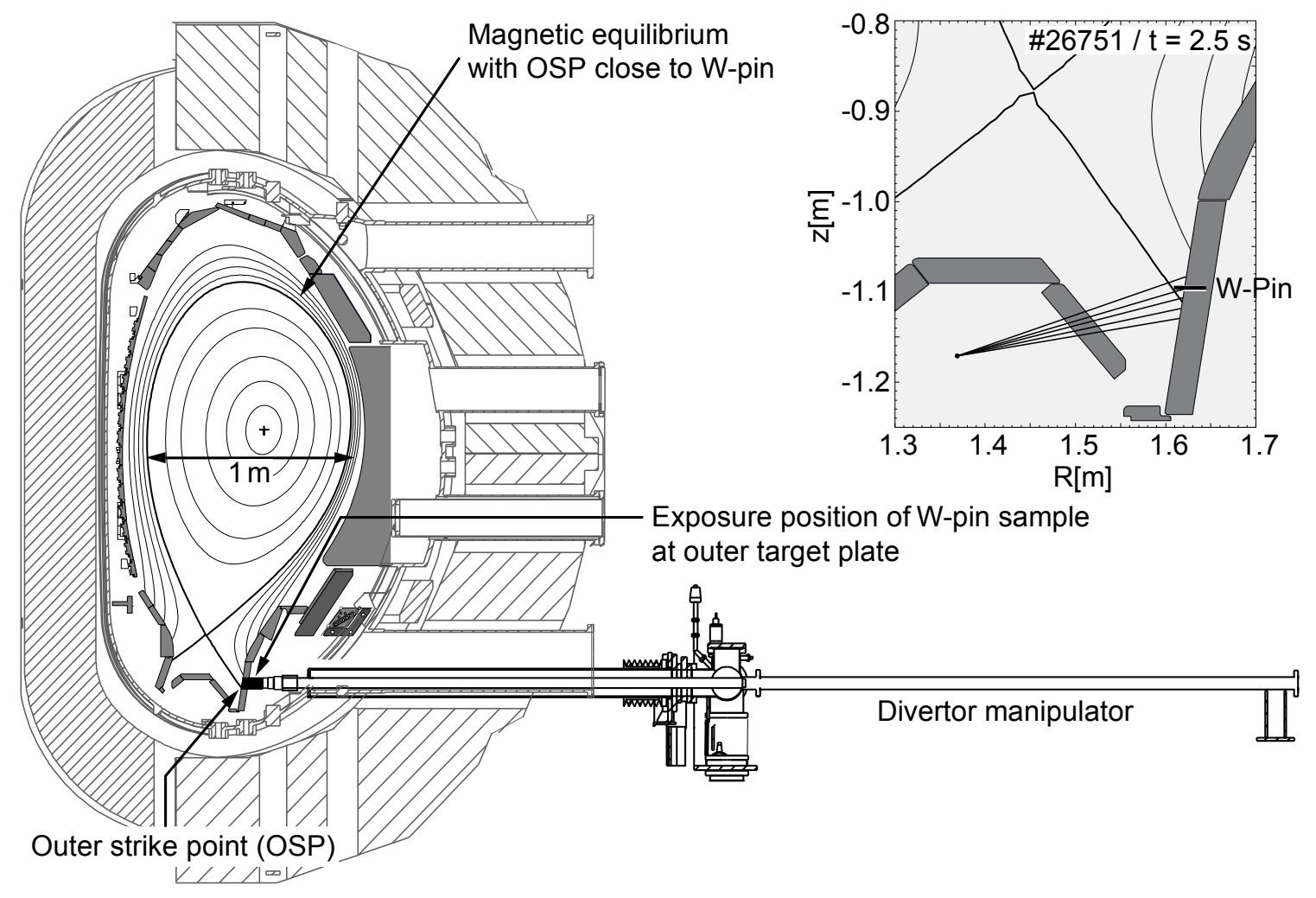


Figure 2

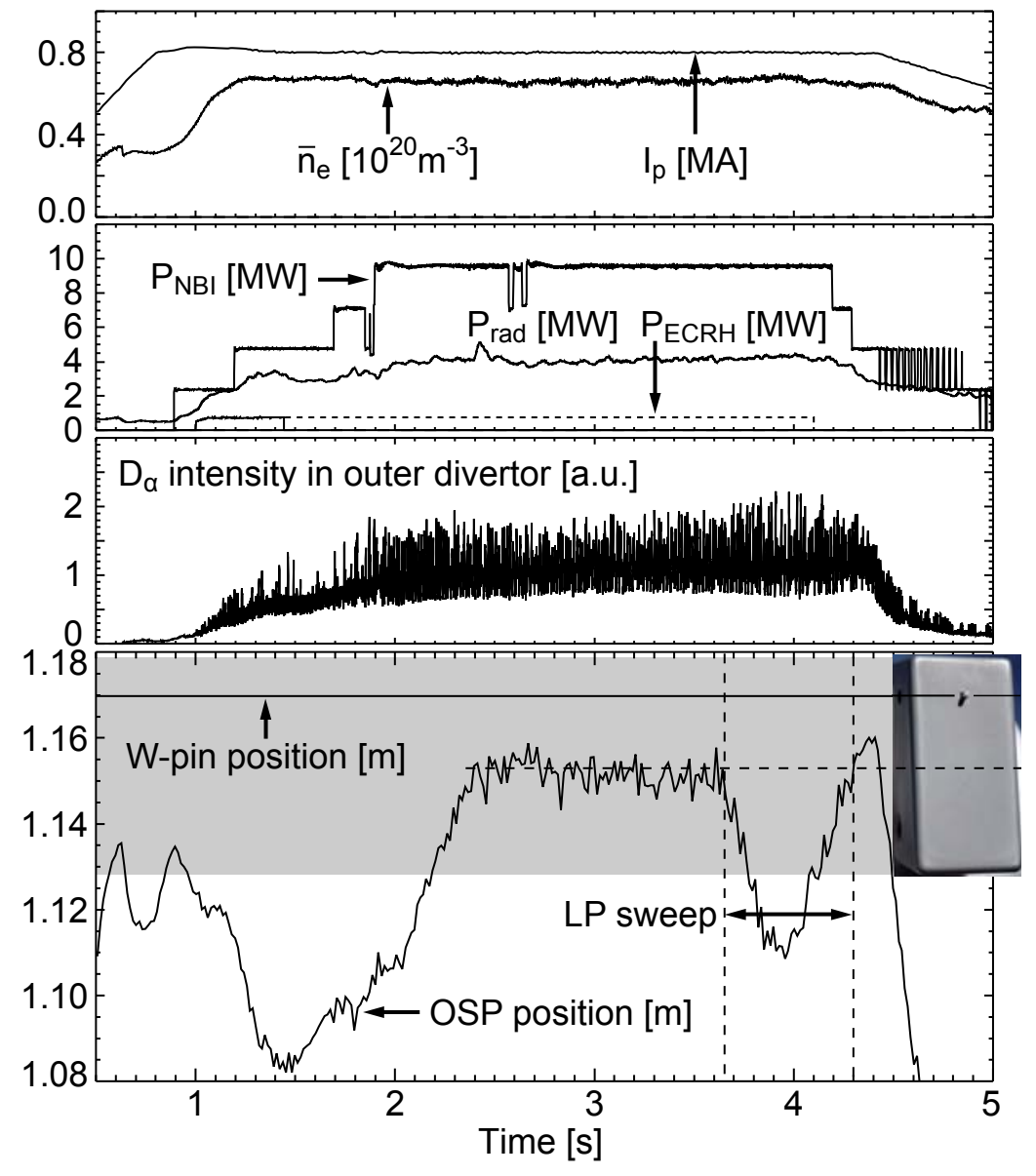


Figure 3

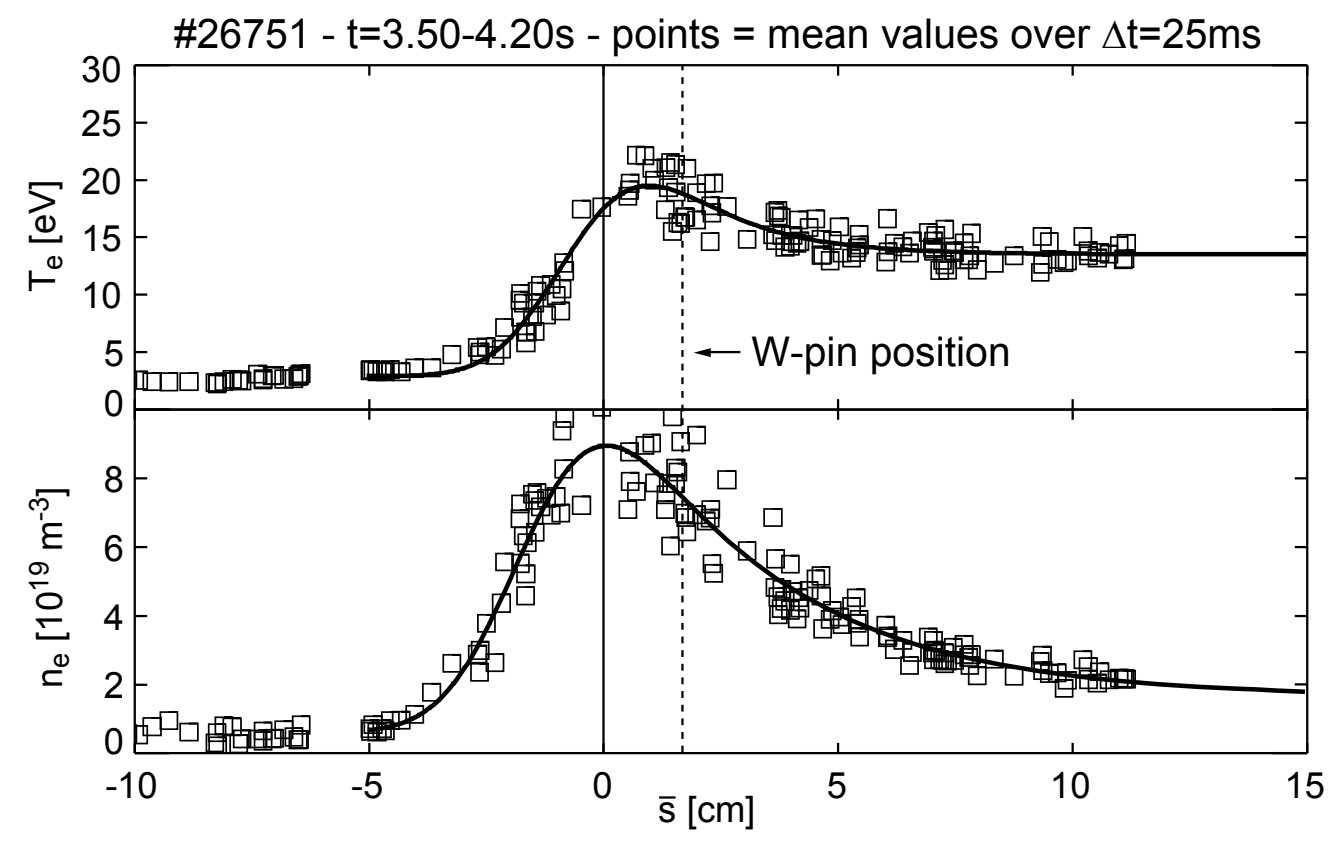


Figure 4
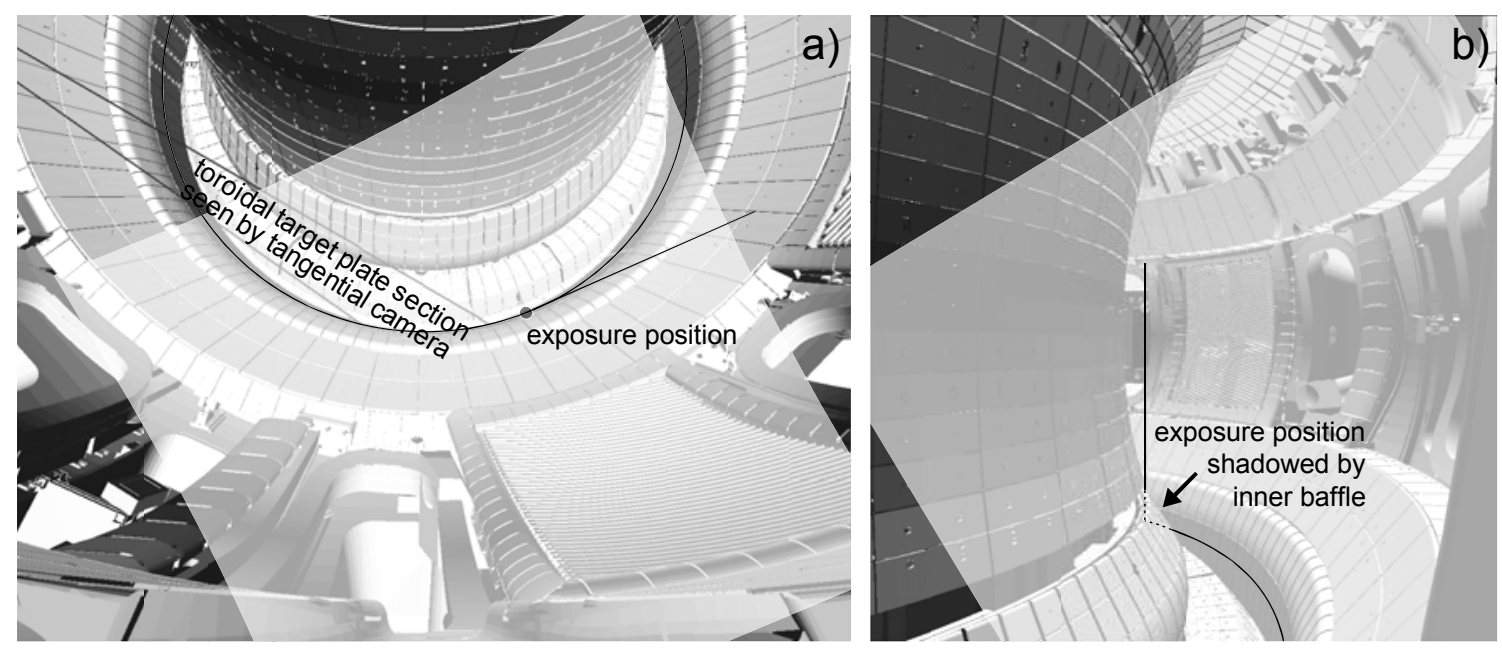
Figure 5
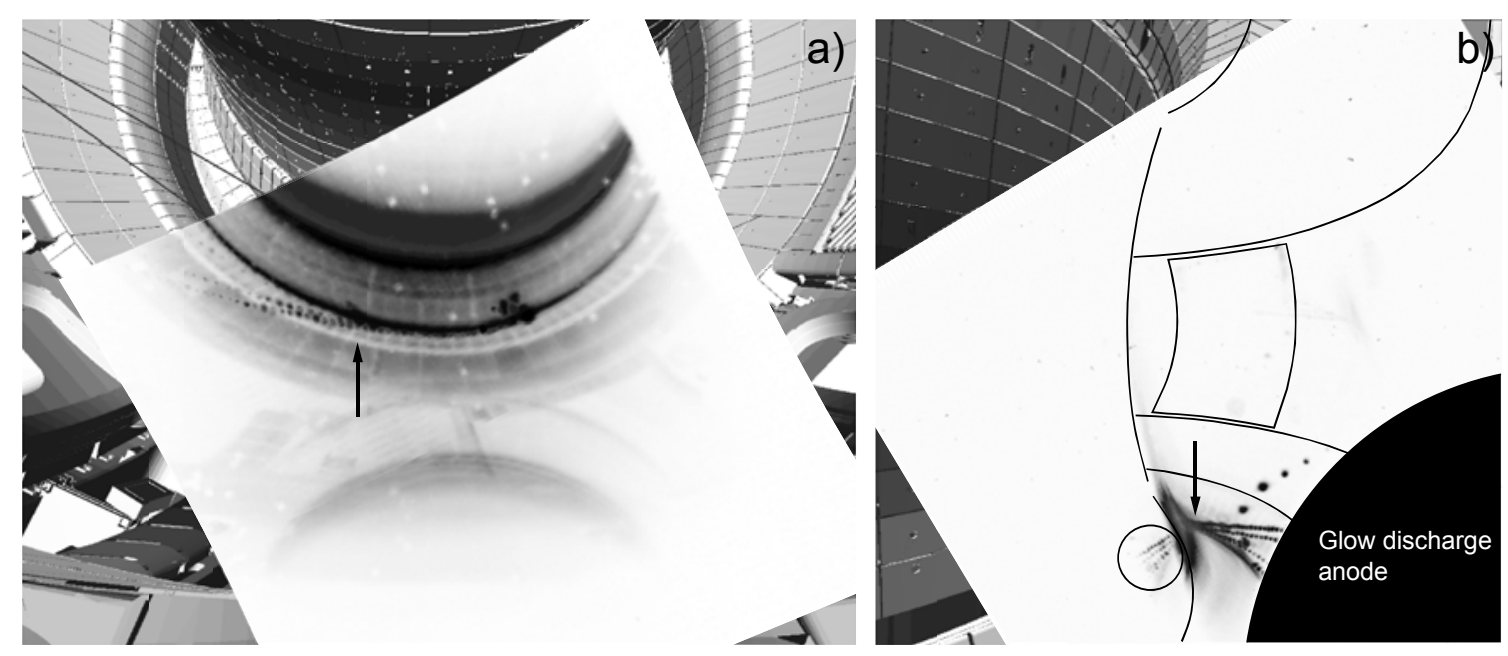
Figure 6

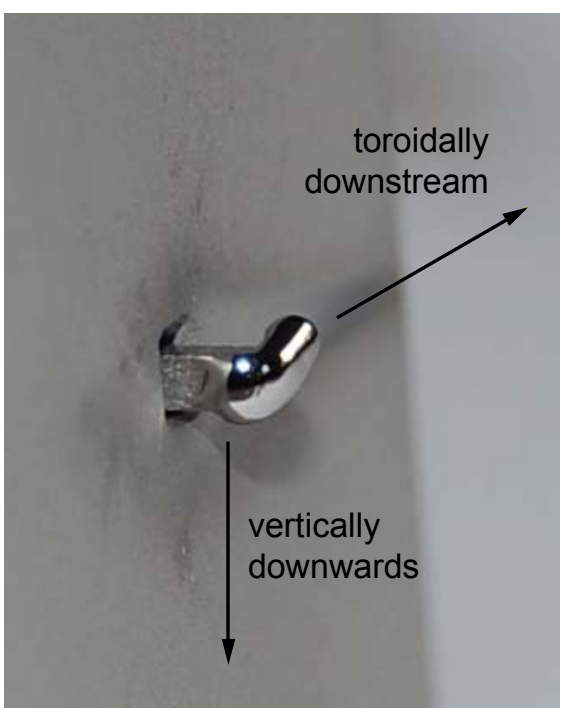


Figure 7
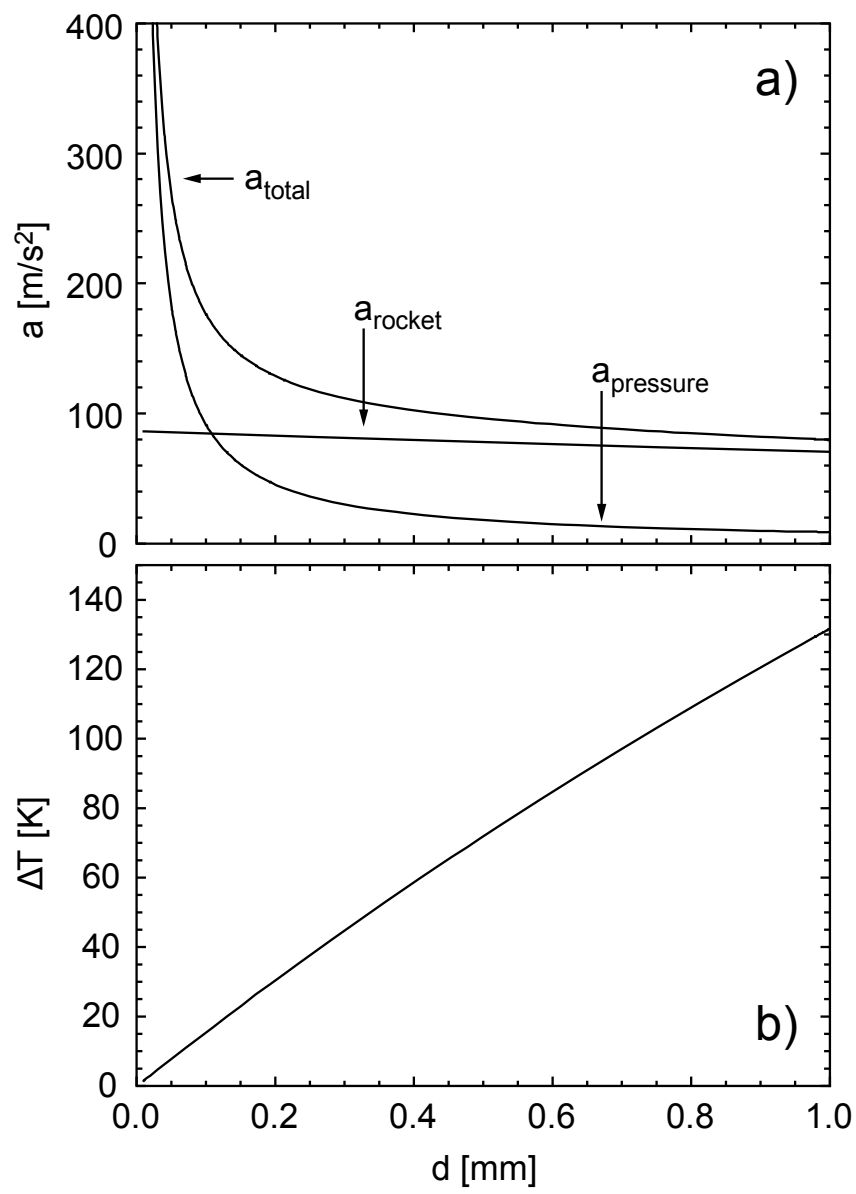
Figure 8

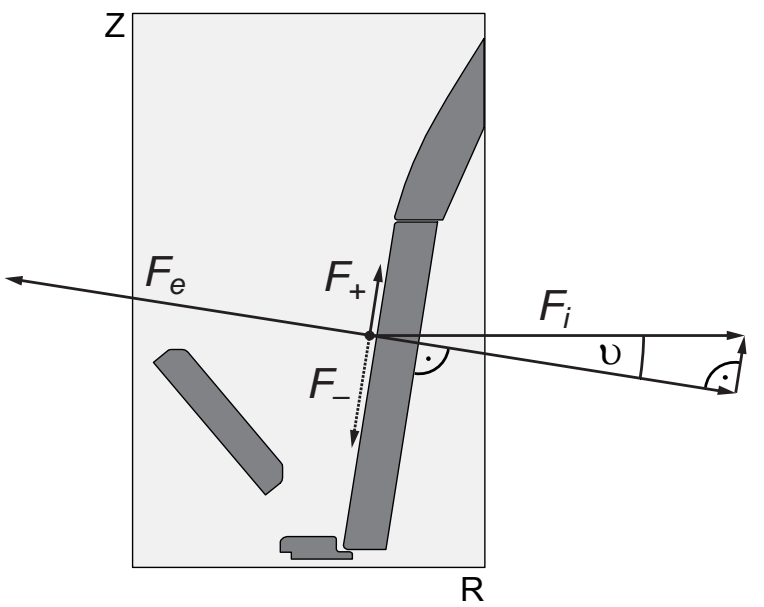


Figure 9
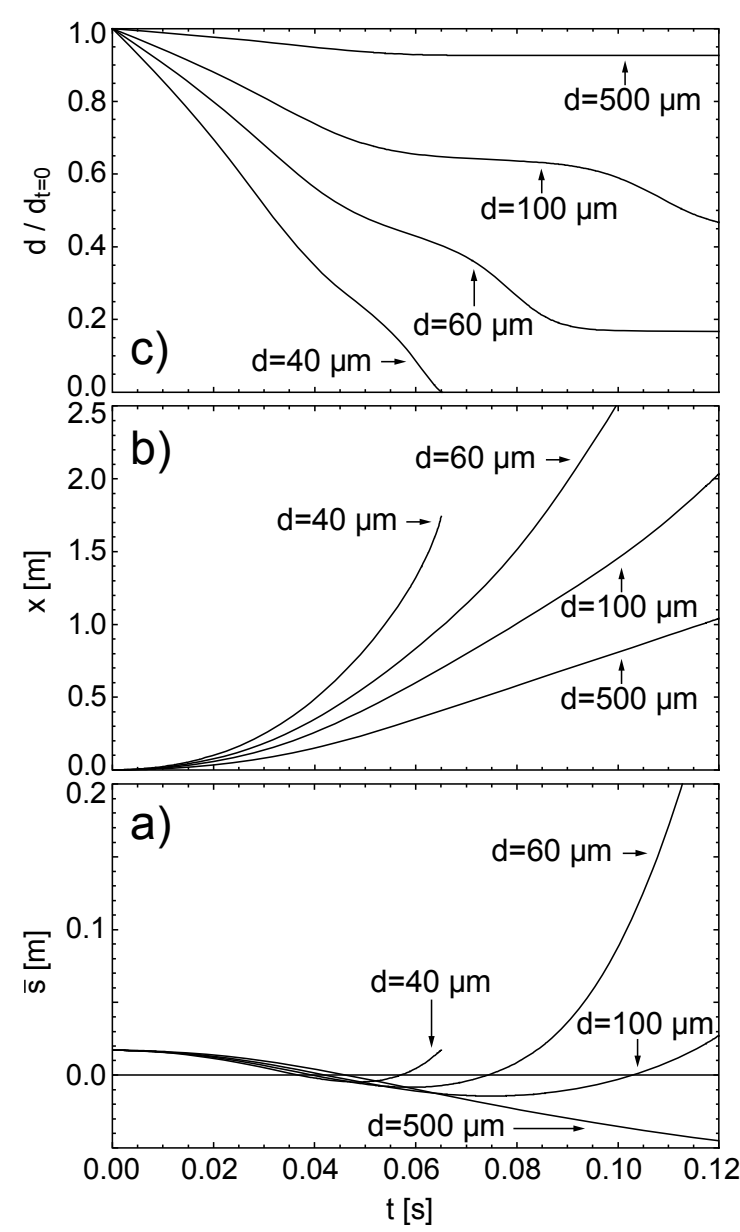
Figure 10

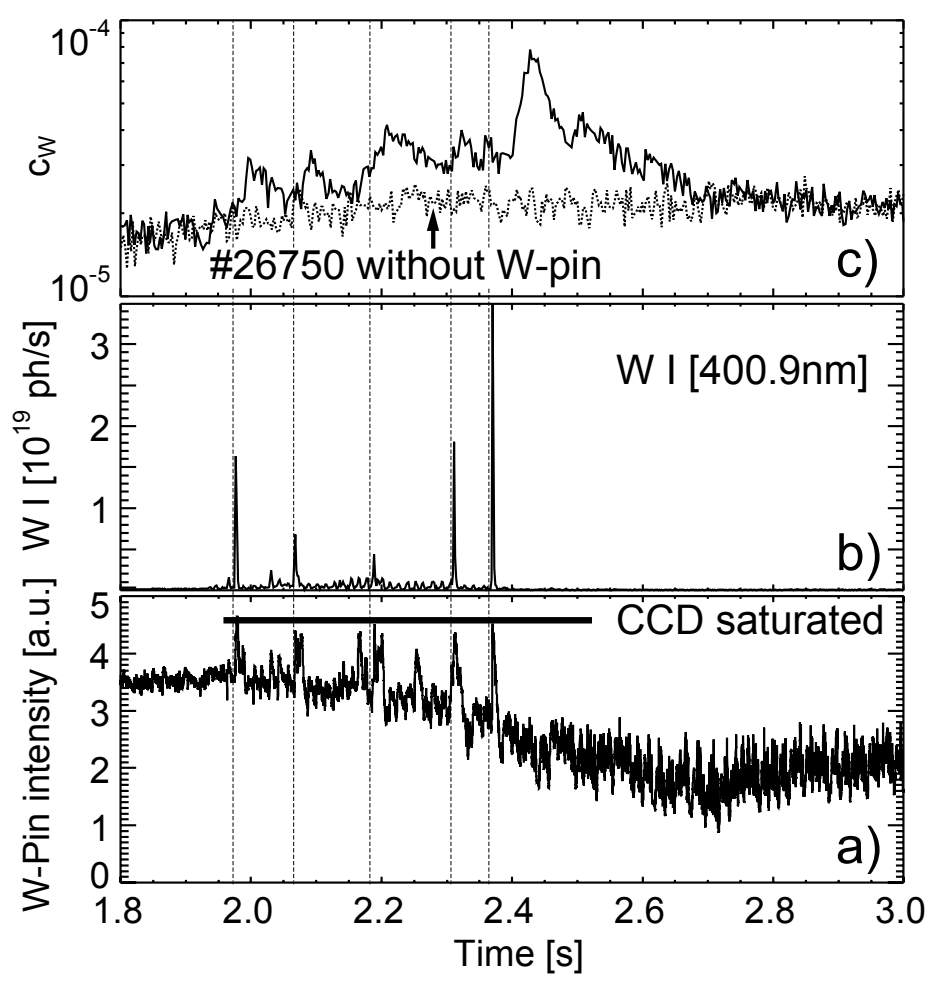

